REVISTA DE METALURGIA, 47 (3)

MAYO-JUNIO, 205-211, 2011

ISSN: 0034-8570

elSSN: 1988-4222

doi: $10.3989 /$ revmetalm.1037

\title{
Coagulación de finos en la flotación de la fluorita ${ }^{(\bullet)}$
}

\author{
P. E. Sarquís*, M. González*, A. Moyano* y V. Bazán**
}

Resumen

Palabras clave

\begin{abstract}
La fluorita es un mineral de mucha importancia en la industria metalúrgica. Se presenta acompañada de silicatos, carbonatos y óxidos. Para la obtención de concentrados comerciales, más de $95 \%$ de $\mathrm{CaF}_{2}$, el mineral se procesa en plantas de flotación donde se usa ácido oleico como colector de la fluorita. Para que el ácido oleico actúe selectivamente es común el uso de reactivos depresores: silicato de sodio, tanino y carbonato de sodio. Estos agentes tienen un efecto dispersante que se evidencia en la separación de sólido-líquido sobre las colas de las plantas. El agua recuperada contiene finos que al ingresar al circuito afectan notoriamente la eficiencia de la flotación. Se estudió el efecto de iones coagulantes para clarificar el agua de retorno. El problema de estos iones está relacionado con sus reacciones con el colector por la formación de compuestos y, posiblemente, sobre la selectividad. Se agregaron cantidades variables de iones $\mathrm{Al}^{3+}, \mathrm{Fe}^{3+}$ y $\mathrm{Ca}^{2+}$ y se evaluó la recuperación de fluorita, la selectividad y en el contenido de sólidos en el agua de los efluentes. Los resultados muestran que algunos iones no solamente no mejoran la calidad del agua sino que además son perjudiciales en la flotación. Otros, por el contrario, contribuyen a reducir el contenido de finos en suspensión. Por lo tanto, y si bien hay una pérdida en la recuperación, este efecto se puede contrarrestar con un pequeño aumento en el consumo de colector.
\end{abstract}

\section{Coagulation of fines in fluorite froth flotation}

\begin{abstract}
Fluorite, a valuable mineral in the metallurgical industry, can be found together with silicates, carbonates and oxides in ore deposits. Commercial concentrates with more than $95 \%$ of $\mathrm{CaF}_{2}$ are obtained processing the mineral in flotation plants and using oleic acid as fluorite collector. Depressor reagents such as sodium silicate, tannin and sodium carbonate are commonly employed to allow the oleic acid selective performance. These reagents cause a dispersant effect separating the solid-liquid solution on tailing plants. Fine particles in the recovered water enter the circuit and affect the flotation efficiency. The effect of coagulant ions was studied as a method to clarify the returned water. The problem of the presence of these ions is related to its reactions with the collector by the formation of compounds and possibly on the selectivity. Variable quantities of $\mathrm{Al}^{3+}, \mathrm{Fe}^{3+}$ and $\mathrm{Ca}^{2+}$ ions were added to the flotation to evaluate its effect on the fluorite recovery, in the selectivity and in solids content in recirculation water. Results show that some ions fail to improve water quality and are detrimental to flotation. On the contrary, some ions contribute to reduce fine content in suspension. Therefore, although there is a recovery reduction, such effect can be counteracted increasing the collector consumption a little.
\end{abstract}

Keywords Flotation; Fluorite; Reagents; Slimes; Coagulation.

\section{INTRODUCCIÓN}

El proceso de producción de concentrados de fluorita se ha ido perfeccionando y evolucionando con los años hacia plantas de flotación donde, luego de una molienda fina, se separan espumas de alta ley. Los esquemas de proceso y los reactivos también tienden a estandarizarse ${ }^{[1]}$.
El ácido oleico es el colector más utilizado. Es un reactivo económico y suficientemente selectivo. Según evidencias de estudios con Espectroscopía con Infrarrojo, el oleico se adsorbe químicamente sobre la fluorita ${ }^{[2}$ y 3]. El PZC de la fluorita es a $\mathrm{pH} 10,0 \mathrm{y}$ se verifica una reacción favorable con el oleico hasta

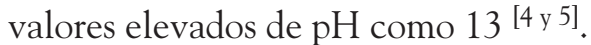

Los minerales acompañantes de la fluorita también

\footnotetext{
(•) Trabajo recibido el día 18 de Junio de 2010 y aceptado en su forma final del día 9 de Octubre 2010.

* Instituto de Investigaciones Mineras, Facultad de Ingeniería. Universidad Nacional de San Juan, Av. Libertador Gral. San Martín 1109 Oeste-5400. San Juan. Argentina. Email: psarquis@unsj.edu.ar.

** CONICET, Instituto de Investigaciones Mineras, Facultad de Ingeniería, Universidad Nacional de San Juan, Av. Libertador Gral. San Martín 1109 -Oeste-5400. San Juan. Argentina. Email: bazan@unsj.edu.ar.
} 
son similares en casi todos los yacimientos; carbonatos, silicatos (mayormente cuarzo) y óxidos. En algunos casos aparecen otros minerales que requieren de procesos más complejos, sobre todo si están presentes pequeñas cantidades de sulfuros de metales básicos, baritina, etc. ${ }^{[6]}$.

Los problemas en la selectividad se relacionan a la presencia de altos contenidos de carbonatos muy activados y de difícil separación de la fluorita ${ }^{[7-9]}$. Otra dificultad la ofrece la ocurrencia de lamas primarias, esto son minerales arcillosos que se desprenden en la molienda y por la simple agitación en medio acuoso. Estas lamas promueven un mayor consumo de reactivos y afectan a la selectividad y a operaciones como la sedimentación y la filtración ${ }^{[10]}$.

La flotación se conduce a $\mathrm{pH}$ alcalino, de 9,5 a 11 regulando el pH con soda Solvay. En el caso de sales semi-solubles como la fluorita en este medio, es inevitable que se produzca un cierto grado de carbonatación ${ }^{[11]}$.

Para reducir la flotabilidad de los silicatos, activados por iones como el calcio, se agrega silicato de sodio que hidrata la superficie y secuestra algunos iones $^{[12]}$. Finalmente, para una mayor selectividad respecto de los carbonatos en la espuma se emplea extracto de quebracho que hidrata a las partículas de carbonatos $^{[13}$ y 14$]$.

Los consumos de estos reactivos se controlan con cuidado debido a que tanto el colector como los depresores no son totalmente selectivos, si hay un exceso de alguno de ellos la calidad de los concentrados se deteriora.

El carbonato de sodio, el silicato de sodio y el tanino tienen un efecto dispersante en la pulpa que en cierta medida ayuda a la selectividad, éste es un efecto positivo ${ }^{[15]}$. Pero por otro lado, el estado de dispersión de las partículas es desventajoso en las operaciones finales de la planta, la sedimentación y

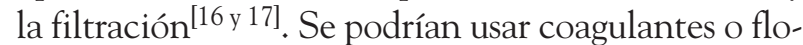
culantes para mejorar la separación sólido-líquido pero se debe tener presente que el agua es un elemento muy escaso en estas operaciones, por lo tanto se recupera y recircula en la molienda. Además las regulaciones ambientales obligan a recuperar los reactivos y el agua de proceso, dejando como efluente un barro de sólidos filtrados que es más fácil de manejar o de darle un destino final como insumo de las cementeras.

La presencia de partículas finas en el agua de proceso es un serio problema en estas plantas. En algunos casos, el mineral se lava con agua en el ingreso a la trituración secundaria utilizando zarandas o clasificadores a espiral. Se descartan los finos con una pérdida significativa de fluorita ${ }^{[10}$ y 18$]$.
El agua recuperada en los espesadores y filtros se envía a la molienda como agua de proceso, es agua turbia con alto contenido de finos en suspensión. El porcentaje de finos en el agua va aumentando hasta un punto que es necesario purgar, eliminando parte del agua de recirculación y reemplazarla por agua fresca.

Considerando este último problema se estudió el agregado de iones coagulantes para facilitar la sedimentación de los finos y mejorar la calidad del agua que se recupera.

El sulfato de aluminio, tanto natural como sintético, es un agente muy usado para el tratamiento del agua potable. Los iones $\mathrm{Al}^{3+}$ neutralizan la carga negativa de las partículas finas y se promueve su coagulación. El sulfato férrico cumple la misma función y en agua potable se admite altos contenidos de sulfato férrico en la compra de insumos coagulantes. Finalmente la cal, es el reactivo universal de plantas de flotación y cianuración como regulador de $\mathrm{pH}$ y como coagulante en los circuitos de lavado en contracorriente ${ }^{[19]}$.

Se estudió el efecto de estos iones como ayudantes de coagulación para reducir el contenido de finos en plantas de flotación de fluorita. Dado que el colector, ácido oleico forma compuestos insolubles con varios cationes, se evaluó también los cambios en la eficiencia en la flotación de la fluorita al aumentar la concentración de iones en el agua.

Los tres iones, $\mathrm{Ca}^{2+}, \mathrm{Fe}^{3+}$ y $\mathrm{Al}^{3+}$, están identificados como activadores de minerales como el cuarzo, feldespato y carbonatos en la interacción con el ácido oleico $^{[19]}$. Este mecanismo de activación está relacionado a la carga superficial de las partículas y permite la formación de compuestos orientados en la superficie con las moléculas de los ácidos grasos y esto reduce la selectividad.

\section{PARTE EXPERIMENTAL}

Para los estudios experimentales se utilizó un mineral de fluorita de un yacimiento de Argentina actualmente en explotación.

Con relación al yacimiento y a su procesamiento industrial se puede decir que la mina se encuentra al sur de la provincia de Córdoba en ambiente de Sierras Pampeanas. Los estudios mineralógicos y los análisis químicos indican que está formado por un $47 \%$ de fluorita, $2 \%$ de carbonatos, $46 \%$ de cuarzo y otros silicatos y $5 \%$ de $\mathrm{R}_{2} \mathrm{O}_{3}$.

En el yacimiento presenta zonas brechosas donde el mineral aparece alterado y con abundante contenido de lamas primarias. Fuera de estos sectores, el mineral en general contiene una cantidad importante de finos que dificultan la flotación en la planta de 
proceso. Cuando se tritura la muestra y se muele en molino de bolas, las partículas ultrafinas (inferiores a $15 \mu \mathrm{m}$ ), representan un $5 \%$ en peso.

En la planta, para atenuar este inconveniente, en el circuito de trituración y clasificación se hace un lavado con agua sobre una zaranda y el fino se trata nuevamente en un clasificador a espiral para recuperar parte de la fluorita fina. A pesar de esto, se elimina en forma directa al dique de colas un producto del lavado donde se pierde un $4 \%$ de la fluorita.

La muestra de mineral recibida en el laboratorio y utilizada en el presente estudio, se trituró a $-2 \mathrm{~mm}$ en seco y luego se hicieron moliendas sobre porciones de $1 \mathrm{~kg}$ en molino de barras antes de cada ensayo de flotación. La molienda fue a 60 \% -74 micrones, $30 \%-44$ micrones.

Los ensayos de flotación se hicieron con muestras de $250 \mathrm{~g}$ en celda de flotación Denver. Primero se acondicionó la pulpa con $400 \mathrm{~g} / \mathrm{t}$ de carbonato de sodio para ajustar el $\mathrm{pH}$ en 9,5, luego se agregaron las sales que aportan los iones coagulantes, las mismas se acondicionaron por $3 \mathrm{~min}$. Posteriormente y en forma secuencial, se agregaron el silicato de sodio, el tanino y finalmente el ácido oleico. Los consumos de estos reactivos fueron de 200, 200 y $500 \mathrm{~g} / \mathrm{t}$, respectivamente. El tiempo de acondicionamiento de los agentes depresores fue de $5 \mathrm{~min}$. En el caso del colector, el tiempo de acondicionamiento fue de 3 min. Luego del acondicionamiento de los reactivos se procedió a la espumación la que se extendió por un tiempo de $7 \mathrm{~min}$.

Los productos se secaron, pesaron y analizaron por vía gravimetría en crisoles de platino. Se tomó una muestra del líquido sobrenadante de las colas y se determinó el contenido residual de sólidos en suspensión. Las mediciones de turbidez sobre estos líquidos no dieron lecturas correctas debido a la coloración que da el tanino al agua.

\section{ANÁLISIS DE RESULTADOS}

\subsection{Efecto del agregado de iones coagulantes}

Se hicieron ensayos de flotación estándar y luego incorporando además cantidades crecientes de sulfato de aluminio, sulfato férrico y de cal. En forma adicional, se realizaron ensayos donde el $\mathrm{pH}$ se reguló en 9,5 con cal en lugar de carbonato de sodio y se agregó los mismos consumos de sulfato de aluminio de la serie anterior.

Esta última serie se realizó teniendo presente que la cal demostró ser el reactivo más eficiente para coagular partículas finas en suspensión. En cuanto al sulfato de aluminio, si bien es un coagulante menos eficaz que la cal, es el reactivo que afecta en menor medida a la recuperación. Los resultados se muestran en la figura 1.

En estas curvas se observa que el sulfato de aluminio produce una ligera disminución en la recuperación, del orden del $5-6 \%$. El resto de los reactivos, en particular la cal, conducen a pérdidas importantes en la recuperación, del orden del 30 - 40 \%.

Los ácidos carboxílicos y sus jabones son bastante activos con relación a los iones alcalino-térreos, sus oleatos se destacan por un muy bajo producto de solubilidad $^{[20]}$. La disminución en la recuperación muestra que parte del ácido oleico está comprometido con la formación de compuestos. En el caso del sulfato de aluminio, esta pérdida no es importante y podría compensarse con un aumento en el consumo.

En la figura 2, se muestran las curvas de las determinaciones del contenido de sólidos en suspensión en el agua de las colas de flotación. Este parámetro es un indicador del grado de dispersión de los sólidos finos y además muestra en forma directa el aporte de lamas que retornan al circuito en el agua recuperada.

Sin el agregado de coagulantes, el agua contiene $15 \mathrm{~kg}$ de sólidos por metro cúbico (1,5\% en peso), lo que se evidencia en una marcada turbidez.

La cal es muy efectiva en la coagulación, en las series de ensayos adonde se agregaron iones calcio, el contenido de finos en suspensión se reduce a niveles de $0,4 \mathrm{~kg} / \mathrm{m}^{3}$ (0,04\% en peso). El sulfato férrico no se muestra como un buen agente para la coagulación. Finalmente, el sulfato de aluminio permite

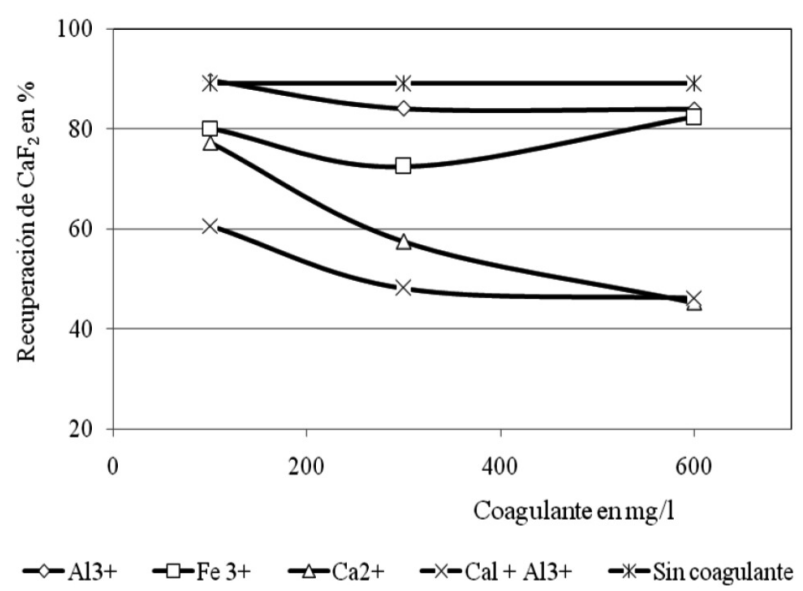

Figura 1. Efecto del agregado de iones coagulantes sobre la recuperación de $\mathrm{CaF}_{2}$. Ensayos de flotación Rougher.

Figure 1. Effect of the use of coagulants ions on $\mathrm{CaF}_{2}$ recovery. Rougher flotation tests. 


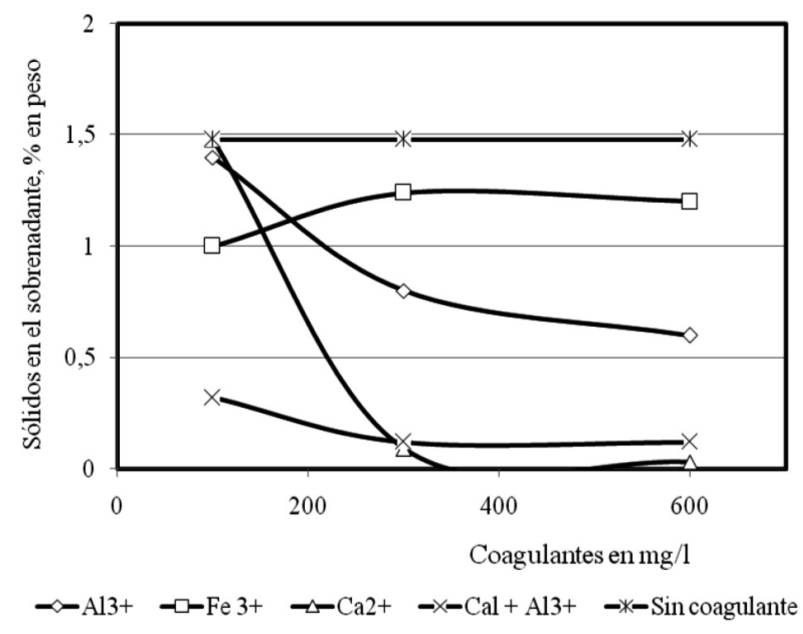

Figura 2. Contenido de sólidos en suspensión en el agua de las colas de flotación. Efecto del agregado de coagulantes.

Figure 2. Solids in suspension in flotation tails water. Effect of coagulant reagents.

sedimentar al $50 \%$ de los finos en suspensión que contiene el agua.

\subsection{Ensayos con agregado de sulfato de aluminio}

En la figura 3, se presentan las curvas de los ensayos con cantidades crecientes de sulfato de aluminio. Al

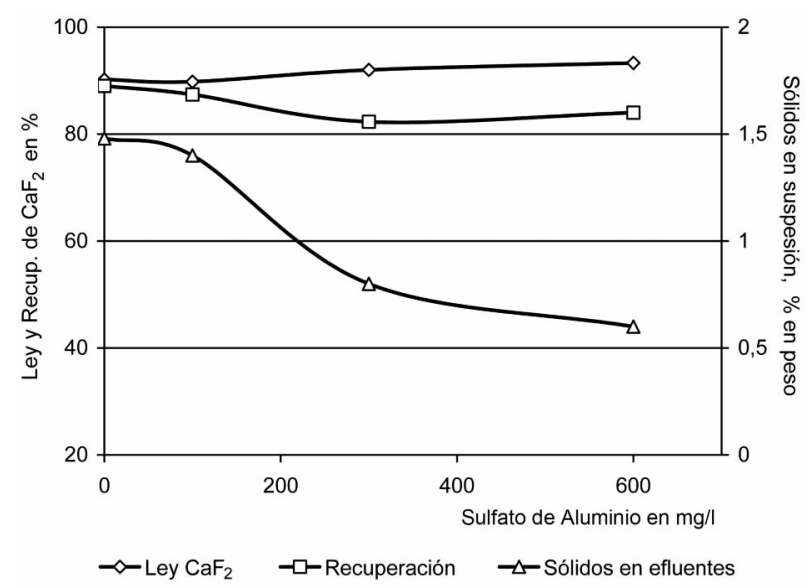

Figura 3. Efecto del agregado de sulfato de aluminio en la recuperación de $\mathrm{CaF}_{2}$, la ley y el contenido de sólidos en suspensión.

Figure 3. Effect of adding aluminum sulfate in $\mathrm{CaF}_{2}$ recovery, in the grade and in the solids in suspension content. aumentar el consumo de coagulante mejora la selectividad, con un aumento en la ley del concentrado de un 2 - $3 \%$. Según trabajos de Mokrousov, V. ${ }^{[21]}$, esto se explica porque la acción depresora del silicato de sodio se ve incrementada con la presencia de sales solubles de aluminio.

Según este estudio, el ión aluminio tiene un efecto depresor sobre los carbonatos pero no afecta a la fluorita. La introducción de este ión en la pulpa promueve una acción más efectiva del silicato de sodio como depresor de los minerales de ganga. Además, este autor comprobó que el ión $\mathrm{Al}^{3+}$ podría promover la flotabilidad de la fluorita.

La recuperación se ve levemente reducida por la presencia de iones $\mathrm{Al}^{3+}$. Pero esta pérdida se puede compensar con un mayor consumo de colector. Ensayos realizados agregando $300 \mathrm{mg} / \mathrm{l} \mathrm{de} \mathrm{sul-}$ fato de aluminio y con un aumento del consumo de ácido oleico del $15 \%$ (575 g/t), demuestran que la pérdida se puede corregir con un mayor consumo de colector.

Con relación a su efecto como coagulante, se observa que con un consumo de $300 \mathrm{~g} / \mathrm{t}$ de sulfato de aluminio se produce la sedimentación del $50 \%$ de los finos en suspensión. Este parece ser el consumo más adecuado para el mineral utilizado para el estudio dado que aun duplicando el gasto no se consigue una reducción significativa en la turbidez del agua.

\subsection{Efecto del agregado de sulfato férrico}

La presencia de iones $\mathrm{Fe}^{3+}$ no es efectiva en la coagulación de las partículas finas en suspensión. El contenido de finos no cambia en forma notoria con el agregado de la sal férrica, figura 4.

Como en el caso del aluminio, el ión férrico parece mejorar la selectividad con un ligero aumento en la ley del concentrado, inferior al obtenido con alumino, pero al aumentar la dosis de $\mathrm{Fe}^{3+}$ la ley disminuye.

La recuperación se ve afectada en un valor inaceptable del 10 \%. Estudios del efecto del ión férrico en flotaciones empleando ácidos carboxílicos como colector, indican que con la presencia de una alta concentración de iones $\mathrm{Fe}^{3+}$ la recuperación se ve disminuida debido que una parte considerable del ácido oleico se gasta en la formación de oleatos polivalentes ${ }^{[20]}$.

Evaluando los tres indicadores se puede concluir que el ión férrico no es adecuado como coagulante de los finos para este tipo de flotaciones. 


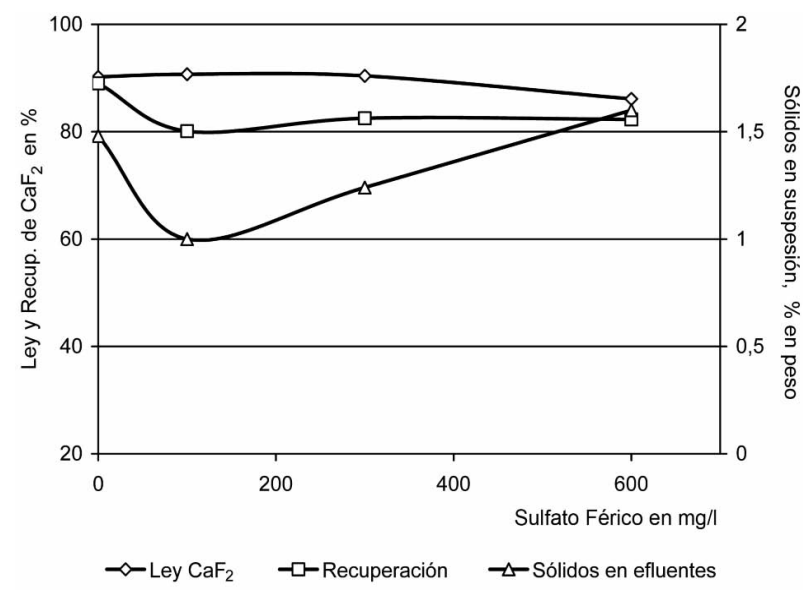

Figura 4. Efecto del agregado de sulfato férrico en la recuperación de $\mathrm{CaF}_{2}$, ley y contenido de sólidos en suspensión.

Figure 4. Effect of adding ferric sulfate in $\mathrm{CaF}_{2}$ recovery, grade and in the solids in suspension content.

\subsection{Efecto del agregado de cal}

La cal produce un drástico descenso en el contenido de sólidos en suspensión llegando a $0,4 \mathrm{~kg} / \mathrm{m}^{3}(0,04 \%$ en peso), pero actúa negativamente en la flotación, figura 5 .
Tanto la ley como la recuperación disminuyen en forma inaceptable para los estándares de la flotación de la fluorita. La presencia de iones calcio promueve la formación de compuestos insolubles con el ácido oleico; oleato de calcio. Por otro lado los iones calcio son fuertes activadores de los silicatos frente a colectores como los ácidos grasos. Por esta razón el cuarzo y otros silicatos se reportan en la espuma y diluyen al concentrado.

La bibliografía señala que la interacción entre el ácido oleico y el cuarzo en ausencia de iones activadores, se realiza en forma no orientada. Por lo que la flotabilidad es muy baja. La presencia en el medio de iones activadores como el $\mathrm{Ca}^{2+}$, induce a la formación de compuestos orientados sobre la superficie, aumentando su presencia en la espuma ${ }^{[15]}$. Esto explica la dilución del concentrado de fluorita.

\subsection{Acondicionamiento con sulfato de aluminio. Ensayos usando cal para ajustar el $\mathrm{pH}$}

Si la pulpa se acondiciona con cal en lugar de carbonato de sodio como regulador del $\mathrm{pH}$ y se hacen agregados de sulfato de aluminio se observa una fuerte reducción del contenido de sólidos en suspensión, figura 6. La acción conjunta de los iones $\mathrm{Al}^{3+}$ y $\mathrm{Ca}^{2+}$ conduce a una neutralización del potencial Zeta de

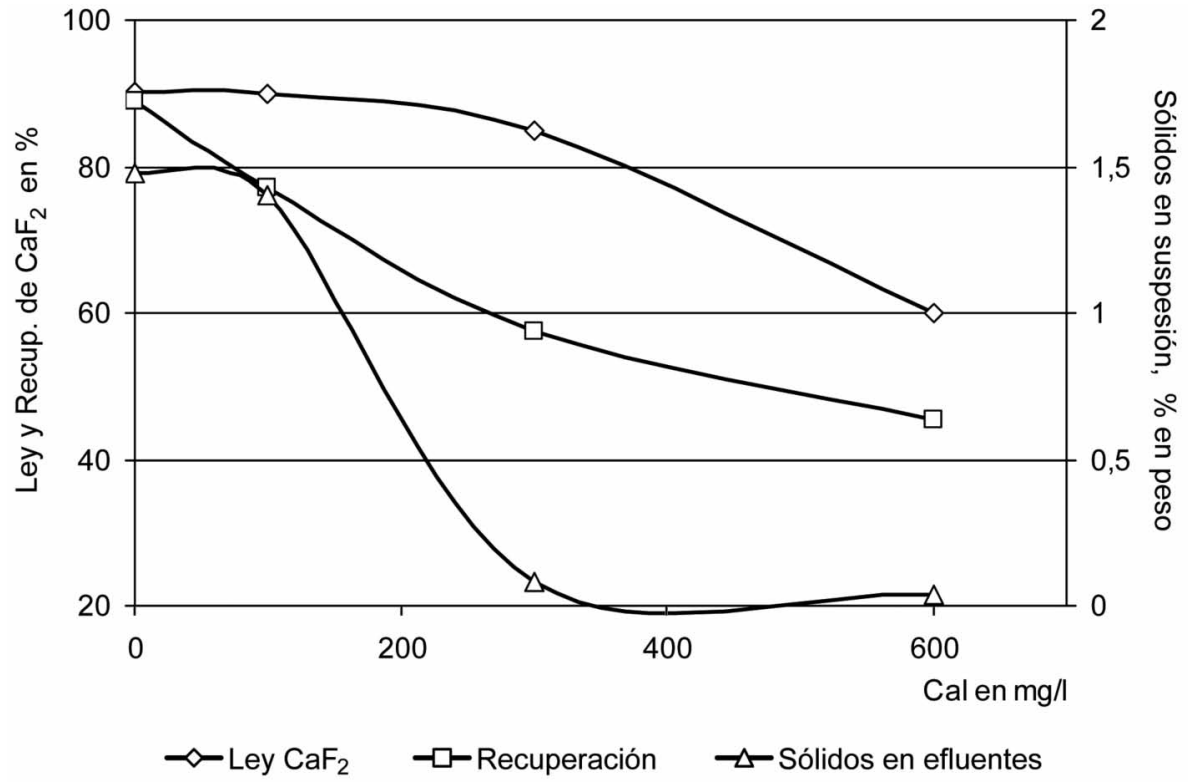

Figura 5. Efecto del agregado de cal en la recuperación de $\mathrm{CaF}_{2}$, ley y contenido de sólidos en suspensión.

Figure 5. Effect of adding lime in $\mathrm{CaF}_{2}$ recovery, grade and in solids content in suspension. 


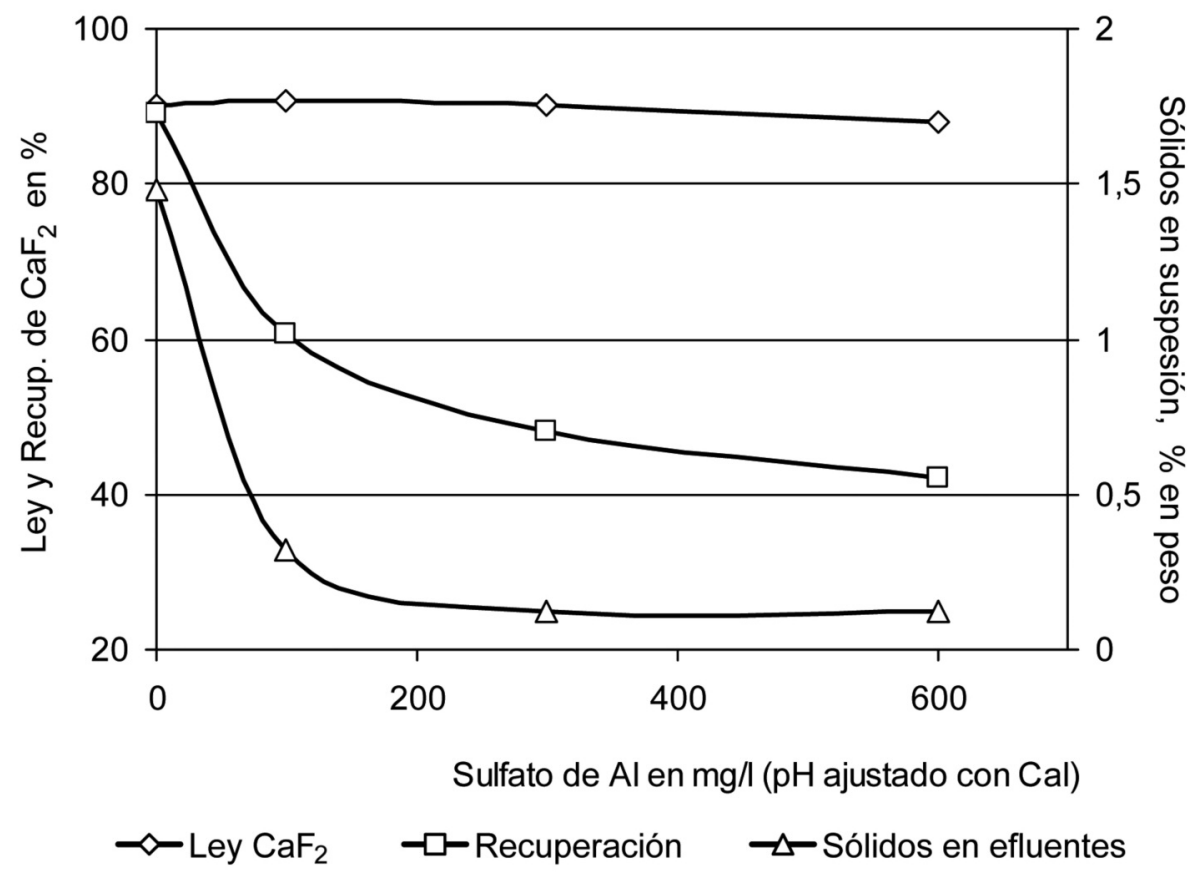

Figura 6. Efecto del agregado de sulfato de aluminio sobre la recuperación de $\mathrm{CaF}_{2}$, ley y contenido de sólidos en suspensión. Ensayos con cal como regulador del $\mathrm{pH}$.

Figure 6. Effect of adding aluminum sulfate in $\mathrm{CaF}_{2}$ recovery, in the grade and the content of solids in suspension. Lime as $\mathrm{pH}$ regulator.

las partículas finas que sedimentan. Mediciones realizadas con un equipo Zeta-Meter, sobre las partículas remanentes en el agua dieron una media de $-22 \mathrm{mV}$.

Analizando las curvas de la ley y recuperación de la figura 6 se observa que al igual de lo que se verifica con los ensayos de la figura 5 , la presencia de cal es inaceptable en esta flotación con ácidos grasos.

\section{CONCLUSIONES}

- El agregado de iones coagulantes en la fórmula de reactivos de la flotación de mineral de fluorita con ácido oleico, permite neutralizar la carga de las partículas finas en suspensión. Los iones calcio y aluminio producen una fuerte reducción del contenido de sólidos en el agua recuperada de las colas de flotación.

- La cal afecta tanto a la recuperación como a la selectividad, lo que la hace inaceptable como coagulante. La formación de compuestos insolubles con el colector y la activación de minerales de ganga son efectos indeseados que no pueden resolverse con cambios en los consumos de reactivos.

- El sulfato férrico no es efectivo como coagulante y por otro lado afecta a la flotación.
- El sulfato de aluminio es en este caso el coagulante más recomendable. Produce una disminución del 50 \% de los finos en suspensión, mejora la selectividad y produce una pequeña pérdida en la recuperación que puede compensarse con un mayor consumo de colector.

\section{REFERENCIAS}

[1] D.R. Nagaraj, Minerals recovery and processing, Ed. Kirk Othmer, Separation Technology, 2nd Edition, EE.UU., 2008, pp. 659-732.

[2] A. Peck, U.S. Bureau of Mines, RI 6202, 1964, pp. 14-16.

[3] K. Marinakis y H. Shergold, Int. J. Miner. Process. 14 (1985) 161-176.

[4] W. Thomas, Mining Chemical Handbook, Ed. Cyted, EE.UU., 2002, pp. 69-130.

[5] R.K. Hanumantha y K. Forssberg, Miner. Eng. 4 (1991) 879-890.

[6] S. K. Mishra, Int. J. Miner. Process. 9 (1982) 59-73.

[7] W. Sampaio y C. Ferreira, VII Meeting of the Southern Hemisphere on mineral Technology, Ouro Preto, Minas Gerais, Brazil, 2007, pp. 243-251. 
[8] H. Schubert, H. Baldauf y W. Kramer, Int. J. Miner. Process. 30 (1990) 185-193.

[9] Y. Zhang y S. Song, Miner. Eng. 16 (2003) 597-600.

[10] P. Sarquís, V. Bazán, Universidad Nacional de San Juan, Informe IIM-023-08. Ed. FUNSJ, UNSJ, Argentina, 2008, pp. 8-11.

[11] J.D. Millar y J.B and Hiskey, Colloid Interface Sci. 41 (1972) 567-573.

[12] M.C. Fuerstenau, G. Gutiérrez y D. Elgillani, Trans. AIME, 242 (1968) 319-320.

[13] G.V. Caesar, Starch and its derivates, Edit. J.A. Radley. Chapman and Hall, London, Inglaterra, 1968, pp. 221-223.
[14] J. Wang y P. Samasundaran, Miner. Eng, 18(2005) 77-81.

[15] M.J. Pearse, Miner. Eng. 18 (2005) 139-149.

[16] S.H. Chiang, Fluid Part. Separ. J. 6 (1993) 64.

[17] P. Somasundaran, Fine Particle Processing, AIME, Vol. 2, New York, EE.UU., 1980, pp. 947

[18] S. Chandler y H. Sis, Miner. Eng. 16 (2003) 577-585.

[19] H. Yuehua y R. Chi, Ind. Eng. Chem. Res. 42 (2003) 1641-1647.

[20] S.V. Dudenhov, Tsvet. Metallurgy 40 (1967) 18-21.

[21] V. A. Mokrousov, Gorny Zhurna 1 (1945) 30-33. 